\title{
Performance Review of Successive Cancellation Decoding Methods of Polar Codes
}

\author{
Chaudhari Sachin $\mathbf{J}^{1}$, Patel Hardik $\mathbf{N}^{2}$ \\ ${ }^{1}$ Post Graduet Student, Parul Institute of Engineering and Technology \\ ${ }^{2}$ Assistant Professor, Parul Institute of Engineering and Technology
}

\begin{abstract}
The first provably capacity achieving codes with low complexity named polar codes were discovered recently and the successive cancellation (SC) decoding is widely known decoding algorithm for polar codes. There are many techniques like folded SC and permuted SC available for improve the SC decoding algorithm. In this paper, we study the different methods of SC decoding for polar codes which are recently developed and give a comparison based on bit error rate (BER) and decoding complexity.
\end{abstract}

Keywords: Polar codes; SC decoding; List Decoding; Decoding Latency; Channel Capacity; BER; SNR; Folded Structure.

\section{Introduction}

Polar codes, the first provable class of channel capacity achieving codes introduced by Arikan [Arikan, 2009] since Shannon presented the noisy channel coding theorem [Shannon, 1948]. The standard SC decoding algorithm presented in [Tal, 2015] have $O(N \log N)$ decoding complexity, where $N$ is the code length. But for achieving channel capacity it requires large size of block length so in that way to reduce block length and hence reduced decoding complexity and improve BER many methods [Li, 2012], [Chan, 2013] and [Haung, 2013] have been introduced.

In this paper some latest methods of SC decoding [Kahraman, 2014], [Vangala, 2014] and [Liu, 2015] are presented and compared for decoding complexity and BER performances.

\section{Background}

Polar Codes

Let, $F=\left[\begin{array}{ll}0 & 1 \\ 1 & 1\end{array}\right], F^{\otimes n} \quad$ is a $N \times N$ matrix, where $N=2^{n}, \otimes n$ denotes the $n$th Kronecker power, and $F^{\otimes n}=F \otimes F^{\otimes(n-1)}$. Let the $n$-bit binary representation of integer $i$ be $b_{n-1}, b_{n-2}, \ldots, b_{0}$. The $n$ - bit binary representation $b_{0}, b_{1}, \ldots b_{n-1}$ is a bit-reversal order of $i$. The generator matrix of polar code is defined as $G_{N}=B_{N} F^{\otimes n}$, where $B_{N}$ is a bit-reversal permutation matrix. The polar code is generated by

$$
x_{1}^{N}=u_{1}^{N} G_{N}=u_{1}^{N} B_{N} F^{\otimes n}
$$

where $x_{1}^{N}=\left(x_{1}, x_{2}, \ldots, x_{n}\right)$ is the encoded bit sequence, and $u_{1}^{N}=\left(u_{1}, u_{2}, \ldots, u_{N}\right)$ is the encoding bit sequence. The bit indexes of $u_{1}^{N}$ are divided into two subsets: the one containing the information bits represented by $A$ and the other containing the frozen bits represented by $A^{c}$. The polar code can be further expressed as

$$
x_{1}^{N}=u_{A} G_{N}(A) \oplus u_{A^{c}} G_{N}\left(A^{c}\right)
$$

Eq. 2

where $G_{N}(A)$ denotes the submatrix of $G_{N}$ formed by the rows with indices in $A$, and $G_{N}\left(A^{c}\right)$ denotes the submatrix of $G_{N}$ formed by the rows with indices in $A^{c}$. $u_{A}$ are the information bits, and $u_{A^{c}}$ are the frozen bits. Polar codes can be decoded with the very efficient SC decoder, which has a decoding complexity of $O(N \log N)$ and can achieve capacity when $N$ is very large.

\section{Conventional SC Decoding}

Consider a polar code with parameters $\left(N, K, A, u_{A^{c}}\right)$ [Arikan, 2009]. where $N, K, u$, and $u_{A^{c}}$ denote the code length, information length, set of information bits, and frozen bit values, respectively. The estimation is $\hat{u}_{1}^{N}=\left(\hat{u}_{1}, \ldots, \hat{u}_{N}\right)$. If $\hat{u}_{i}$ is a frozen bit, $\widehat{u}_{i}=0$. Otherwise, if $u_{i}$ is an information bit, then

$$
\widehat{u}_{i}=\left\{\begin{array}{l}
0, i f W_{n}^{(i)}\left(y_{1}^{N}, \widehat{u}_{1}^{i-1} / 0\right) \geq W_{N}^{i}\left(y_{1}^{N}, \widehat{u}_{1}^{i-1} / 1\right), \\
1, i f W_{n}^{(i)}\left(y_{1}^{N}, \widehat{u}_{1}^{i-1} / 0\right)<W_{N}^{i}\left(y_{1}^{N}, \widehat{u}_{1}^{i-1} / 1\right),
\end{array} \text { Eq. } 3\right.
$$

Where $W_{N}^{i}\left(y_{1}^{N}, \widehat{u}_{1}^{i-1}\right)$ is the channel transition probability or likelihood probability. For better robust-ness and lower complexity, S decoder over logarithm domain is more preferred. Define the log likelihood probability as

$$
L_{N}^{(i)}\left(y_{1}^{N}, \widehat{u}_{1}^{i-1} / u_{i}\right) \square \ln W_{N}^{(i)}\left(y_{1}^{N}, \widehat{u}_{1}^{i-1} / u_{i}\right)
$$

Eq. 4

\section{Conventional SC List Decoder}

For each step of SC decoder, only the most likely bit decision survived. Whenever certain bit is incorrectly 


\section{International Journal of Science and Research (IJSR) \\ ISSN (Online): 2319-7064}

Index Copernicus Value (2013): 6.14 | Impact Factor (2015): 6.391

decoded, the corresponding decoding fails. In order to deal with this error propagation, an improved SC decoder, named SCL decoder, is proposed. SCL decoder can be regarded as a breadth first search (BFS) version of SC decoder. Similar to $K$-best detection for multi-input multi-output (MIMO) systems, SCL decoder expands and selects paths level-bylevel on the full binary-tree. At each level, SCL decoder expands paths and computes path metrics, then selects the $l$ paths with largest metrics instead of only keeping the best path. In addition, the list of $l$ candidate paths $P \subseteq[G F(2)]^{N \times 2 l}$, are stored for further processing. At the leaf nodes, the path with largest matric wins. The decoded code is then output. Details of SCL decoder are listed in algorithm 1 .

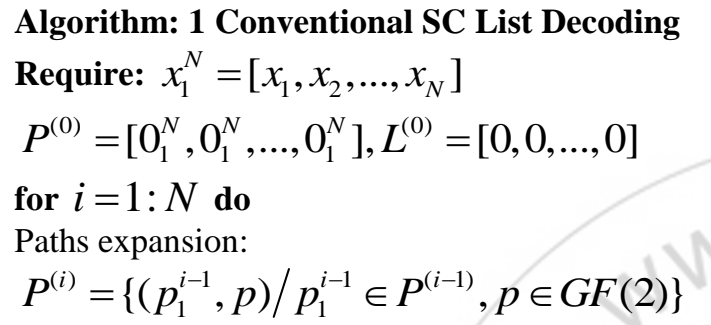

Metric update: iterative computation if $\left|P^{(i)}\right|<=L$ then Continue else

Keep the $l$ candidate paths with largest metric end if

if $i=N$ then

$\widehat{u}_{1}^{N}=\arg \max { }_{p_{1}^{N} \in P}\left(L^{(N)}\right), L^{(N)} \in L^{(N)}$

end if

end for

Fig. 1 illustrates the search process of SCL decoder with $l=2$. It is noted that the dominated computational complexity of each level lies in the $2 l$ path metric comparisons. Therefore, although larger $l$ provides better performance, the increasing complexity makes SCL decoder with large $l$ less favorable.

\section{Different SC Decoding Methods}

\section{Folded SC Decoder}

SC is a suboptimal decoder so it has quasi-linear complexity $N(1+\log N)$ in the code length N. In [Kahraman, 2014], a new non-binary SC decoder with low complexity $\frac{N}{2}\left(1+\log \frac{N}{2}\right)$ was proposed. It named as folded SC decoder. This method is based on folded decoding tree structure, given in [Kahraman, 2013] to implement an efficient ML decoder of polar codes. By applying once the folding operation, a conventional SC decoder can be redesigned as a non-binary half-length SC decoder. In this method conditional probabilities of bit-pairs in $1+\log \frac{N}{2}$ steps of the folded SC decoder were considered instead of the likelihood ratios used in the $1+\log N$ steps of conventional SC decoder. In addition the folding operation enables to construct $\log N$ alternative pairings of bits, which giver the better error performance with the same complexity.

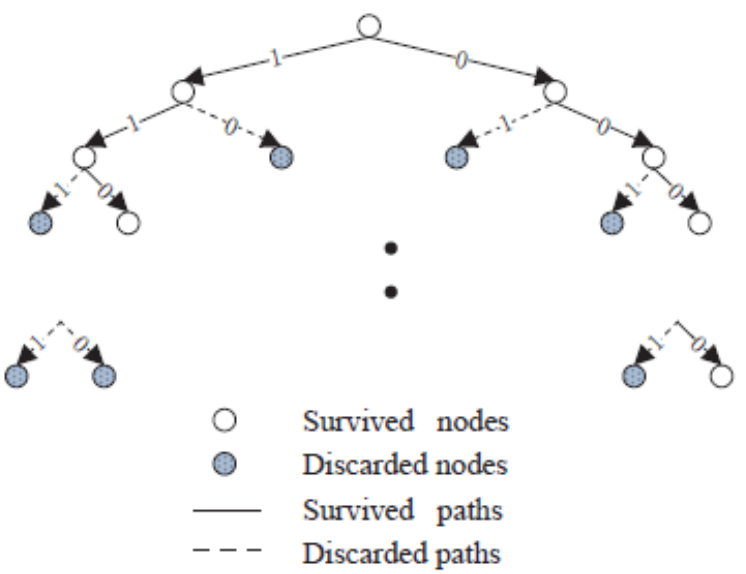

Figure 1: Search process of conventional SCL polar decoder with $l=2$.

\section{Improved Multiple Folded SC Decoder}

Folding is a technique to modify the decoder graph of polar codes based on successive cancellation decoding (SCD). In previous method [Kahraman, 2014] shows folding only for $k \leq 3$ due to rapidly increasing complexity. In this method [Vangala, 2014], an alternative implementation of the multiple-folding SC decoder proposed, to significantly reduce its complexity. The complexity of this algorithm is only slightly higher than that of the original SCD for polar codes. Hence, the algorithm enables to decode longer codes with larger $k \mathrm{~s}$, which exhibit significant performance gains, in addition to the latency gain.

\section{A Stage-Reduced Low-Latency SC Decoder}

In today's world the large code length required by practical applications cause high decoding latency because the conventional SC decoder decodes bits serially. In this method [Liu, 2015] an improved SC decoding method is given which achieves almost $50 \%$ latency reduction with no performance degradation.

This method eliminates the last stage of the conventional SC decoder with a hard decision module. This decoder can reduce the decoding latency by $N$ clock cycles without performance degradation. A simple 1-stage reduced decoding algorithm is given below.

Algorithm 2: The 1-Stage-Reduced Decoding
Algorithm
for $i=1: N / 2$
case 1: $2 i-1 \in A^{c} \& \& 2 i \in A^{c}$
then $\widehat{u}_{2 i-1}=0, \widehat{u}_{2 i}=0$
case 2: $2 i-1 \in A^{c} \& \& 2 i \in A$




\section{International Journal of Science and Research (IJSR) \\ ISSN (Online): 2319-7064}

Index Copernicus Value (2013): 6.14 | Impact Factor (2015): 6.391

then $\widehat{u}_{2 i-1}=0$,

$\widehat{u}_{2 i}=\left\{\begin{array}{l}0, \text { ifL } L_{N / 2}^{(i)}\left(y_{1}^{N / 2}, \hat{a}_{1}^{i-1}\right)+L_{N / 2}^{(i)}\left(y_{N / 2+1}^{N}, \widehat{b}_{1}^{i-1}\right) \geq 0 \\ 1, \text { else }\end{array}\right.$

case 3: $2 i-1 \in A \& \& 2 i \in A^{c}$

then $\hat{u}_{2 i}=0$,

$\hat{u}_{2 i-1}=\left\{\begin{array}{l}0, \text { if } \operatorname{sgn}\left[L_{N / 2}^{(i)}\left(y_{1}^{N / 2}, \hat{a}_{1}^{i-1}\right)\right] \operatorname{sgn}\left[L_{N / 2}^{(i)}\left(y_{N / 2+1}^{N}, \hat{b}_{1}^{i-1}\right)\right] \geq 0 \\ 1, \text { else }\end{array}\right.$

case 4: $2 i-1 \in A \& \& 2 i \in A$

then

$\hat{u}_{2 i-1}=\left\{\begin{array}{l}0, i f \operatorname{sgn}\left[L_{N / 2}^{(i)}\left(y_{1}^{N / 2}, \hat{a}_{1}^{i-1}\right)\right] \operatorname{sgn}\left[L_{N / 2}^{(i)}\left(y_{N / 2+1}^{N}, \hat{b}_{1}^{i-1}\right)\right] \geq 0 \\ 1, \text { else }\end{array}\right.$

$\widehat{u}_{2 i}=\left\{\begin{array}{l}0, i f L_{N / 2}^{(i)}\left(y_{N / 2+1}^{N}, \widehat{b}_{1}^{i-1}\right) \geq 0 \\ 1, \text { else }\end{array}\right.$

end case

$\widehat{a}_{i}=\widehat{u}_{2 i-1} \oplus \widehat{u}_{2 i}, \widehat{b}_{i}=\widehat{u}_{2 i}$

end for

\section{Results}

In this section we give the simulated results of these three $\mathrm{SC}$ decoding methods described in previous section for BER performance. Also give the complexity comparison of that three SC decoding methods.

Fig. 2 shows the BER performance of SC and folded SC decoder for polar code $(128,64)$ at rate 0.5 and $E_{b} / N_{0}=1$. We can see that the BER performance of folded SC decoder is way better than conventional SC decoder. In paper [Kahraman, 2014] more results are shown. In fig. 3 the performance of the multiple folded SC decoder is shown. Fig. 4 shows the performance of 2-stage reduced $\mathrm{SC}$ decoder. For folded SC polar decoding of code length $\mathrm{N}$ $=8192$ and rate $\mathrm{R}=0.8$ considered and Additive White Gaussian Noise (AWGN) channel and BPSK modulation taken. For stage reduced SC decoder code length $\mathrm{N}=1024$ and half rate considered.

Folded SC gives approax $10^{-5}$ BER at $5 \mathrm{~dB}$ SNR. Improved multiple folded SC gives $10^{-5}$ BER at $9.7 \mathrm{~dB}$ SNR and stage reduced SC gives approax $10^{-5}$ BER at 3.5 dB SNR. We can see from the results that in all methods BER performance is better than the conventional SC decoding method also decoding complexity is reduced. These methods are applicable at

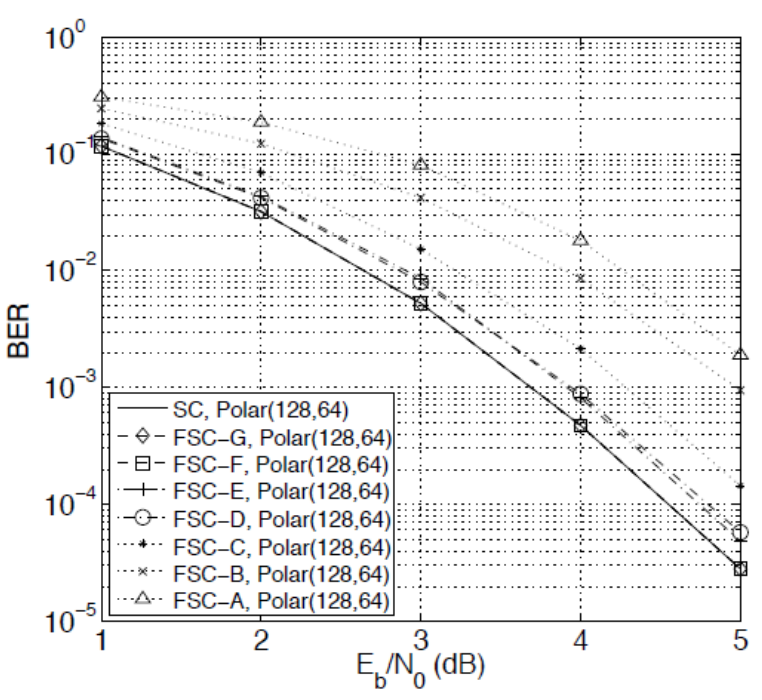

Figure 2: BER performance of SC and folded SC for polar code $(128,64)$

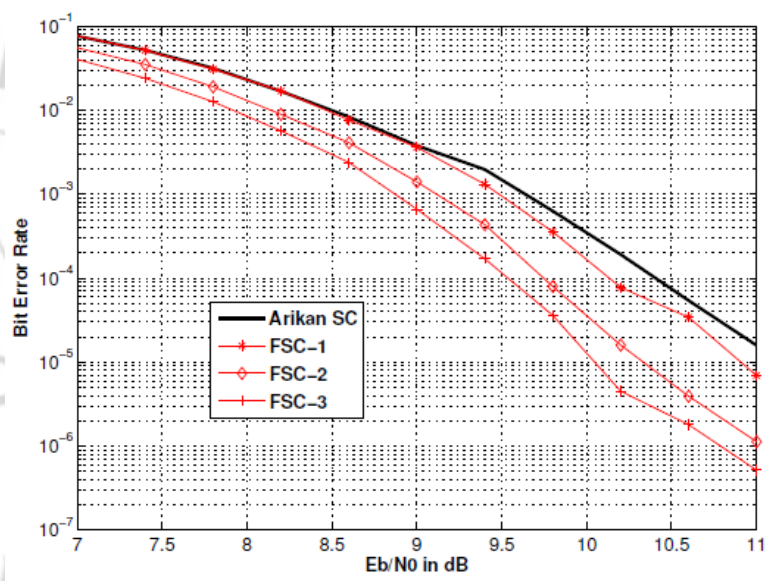

Figure 3: BER performance of SC and multiple folded SC for polar code

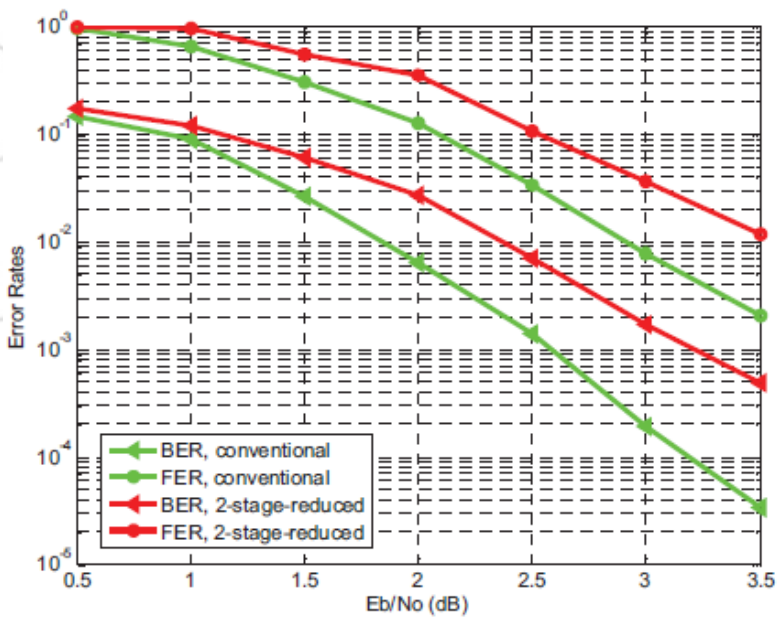

Figure 4: BER and FER performance of SC and 2-stage reduced SC for polar codes

Different applications as per required block length, i.e. for short block length codes folded SC is useful and large code length stage reduced SC is very handy.

Also here we mansion complexity view of these decoding methods. These three methods have reduced the decoding complexity than the conventional SC decoding method. 


\section{International Journal of Science and Research (IJSR) \\ ISSN (Online): 2319-7064}

Index Copernicus Value (2013): 6.14 | Impact Factor (2015): 6.391

Decoding complexity of conventional SC decoder is $O(N \log N)$ where as decoding complexities of folded, improved multiple folded and Stage reduced SC decoders are slight less than the conventional SC decoder. In addition decoding latency of stage reduced SC decoder is $50 \%$ less than conventional SC decoder.

\section{Conclusion}

In this brief, the overview of recent SC decoding methods is given and comparison of these methods with conventional SC decoding method is described on the basis of their bit error rate, decoding complexity and decoding latency. In short all these methods are better than the Conventional SC decoding method in all aspects i.e. BER, complexity and decoding latency. Furthermore we can conclude from this short brief that all these methods are best in their applications i.e. short block length or large block length.

\section{References}

[1] Arikan, E. 2009. Channel Polarization: A Method for Constructing Capacity-Achieving Codes for Symmetric Binary-Input Memoryless Channels. IEEE Transactions on Information Theory, 55, 7 (Jul. 2009), 3051-3073.

[2] Chen, K., Niu, K. and Lin, J. 2013. Improved Successive Cancellation Decoding of Polar Codes. IEEE Transactions on Communications, 61, 8 (Aug. 2013), 3100-3107.

[3] Kahraman, S., Viterbo, E. and Celebi, M.E. 2014. Folded successive cancelation decoding of polar codes. 2014 Australian Communications Theory Workshop (AusCTW), (Feb. 2014), 57-61.

[4] Huang, Diao, Dai, Wu and Chen. An Improvement of Modified Successive-Cancellation Decoder for Polar Codes. IEEE Communications Letters, 17, 12 (December 2013), 2360-2363.

[5] Li, Shen and Tse 2012. An Adaptive Successive Cancellation List Decoder for Polar Codes With Cyclic Redudency Check. IEEE Communication Letters. 16, 12 (December 2012), 2044-2047.

[6] Liu, X., Sha, J., Zhang, C. and Wang, Z. 2015. A stagereduced low-latency successive cancellation decoder for polar codes. 2015 IEEE International Conference on Digital Signal Processing (DSP), (Jul. 2015), 258-262.

[7] Shannon, C.E. 1948. A mathematical theory of communication. The Bell System Technical Journal, 27, 4 (Oct. 1948), 623-656.

[8] Tal, I. and Vardy, A. 2015. List Decoding of Polar Codes. IEEE Transactions on Information Theory, 61, 5 (May. 2015), 2213-2226.

[9] Vangala, H., Viterbo, E. and Hong, Y. 2014. Improved multiple folded successive cancellation decoder for polar codes. 2014 XXXIth URSI General Assembly and Scientific Symposium (URSI GASS), (Aug. 2014), 1-4. 